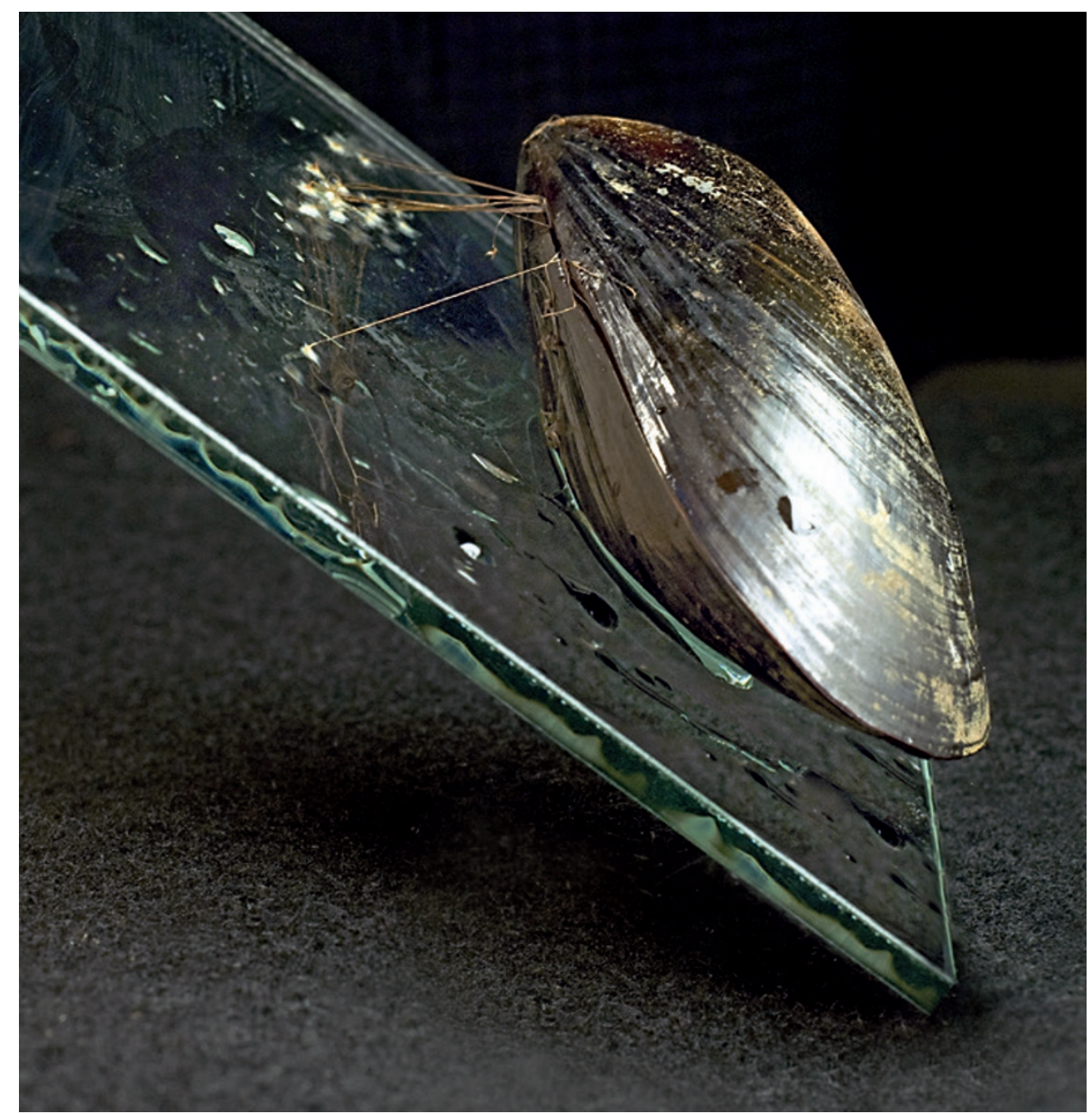

Mussels' adhesive threads are inspiring scientists to create glues that can form bonds underwater.

POLYMERS

\title{
Secrets from the deep sea
}

\section{The mechanism used by mussels to stick to slippery rocks is the idea behind glue that could mend broken bones.}

\section{BY ANDREW R. SCOTT}

$\mathrm{F}$ ifteen years ago, while being thrown about by heavy seas during a scuba dive off the California coast, Jonathan Wilker was impressed by the mussels sticking firm and untroubled to the rocks surounding him. Wilker, a chemist and materials engineer, wondered how the molluscs managed to hold on. In particular, he thought about how the mussels could form a strong adhesive bond under water, given that most artificial glues require completely dry surfaces before application. The thought inspired him to embark on a long journey into the world of marine made much stronger ones. Some of the bio mimetic polymer glues the researchers have developed are now ready for production and commercialization. One of Wilker's particular interests is in using glues to repair wounds. When giving presentations about the medical potential of his work he often asks, "Wouldn't it be better if we could just glue you back together and if we could put sutures out of business?" Speaking from his office, he says that he hopes to find a bonding material that could fix a broken bone and is stronger than the bone itself. That explains the noise drifting into his office from a saw cutting through bones in another room. Pausing to close the door, he apologizes for the "kind of gross" sound and then continues enthusiastically about the possibilities for his wet-setting glues that incorporate ideas culled from mussels and oysters.

\section{MUSSEL-PROTEIN MIMICRY}

The secret to mussel glues is their unusual protein molecules. Specifically, many of the tyrosine amino acids have been converted into 3,4-dihydroxyphenylalanine (DOPA) groups by adding extra hydroxyl $(\mathrm{OH})$ groups to the tyrosine six-carbon rings that protrude from the protein chain. This modification allows crosslinks to form between the protein chains that allow the glue to set. Wilker's laboratory found that naturally occurring iron also seems to be involved, creating an organic-inorganic glue that is not yet fully understood.

Why not extract mussel or oyster glues directly from the animals? Wilker says that is not feasible: each animal makes only tiny amounts of adhesive and so large-scale harvesting is not possible. Wilker's solution to this problem uses a synthetic polymer based on a polystyrene that has been modified in the laboratory to mimic the long chains of mussel proteins, with DOPA-like groups attached to duplicate the wet-setting crosslinking abilities of the mussel glues. The resulting polymers are then combined with simpler chemicals such as iron, which is found in natural mussel glues, to create a range of adhesive options ${ }^{1}$.

"We now have adhesives that, under some circumstances, are stronger than superglue," says Wilker. Medical applications will require rigorous safety and biocompatibility testing. In a promising first step, experiments in late 2014 have found no toxicity to growing cells. The glues are now being trialled for bonding soft tissues and broken bones in the laboratory. Domestic and industrial applications such as adhesives for buildings, vehicles or even false fingernails could be approved more quickly than those for clinical uses; with this in mind, Wilker applied for a patent for the new polymeric adhesives, which was issued in 2014. "We just need to hook up with the right industrial partner now," says Wilker, adding that he is currently sending samples of his glues to a variety of companies for testing. If the results are 
as positive as Wilker hopes, artificial versions of mussel glue may soon make their way into industry and eventually medicine.

\section{THE SENSITIVITY OF SEA CUCUMBERS}

But it is not only mussels and oysters that have piqued the interest of materials scientists. Sea cucumbers have evolved different protective features that researchers are trying to exploit. When threatened by predators, some species of the ocean-floor dwelling, soft-bodied animals can voluntarily stiffen in less than a second so that they become less appealing to prey. Sea cucumbers are able to make the reversible transformation because they possess a natural material that contains fibrils of the protein collagen mixed with a soft matrix. Regulatory protein molecules in the matrix can change the number of cross-links between the collagen fibrils so that the creature is able to switch rapidly between its soft and stiff states.

Jeffrey Capadona and colleagues realized that such variable flexibility might be exactly what they needed to make better microelectrodes for brain implants. Capadona is a biomaterials scientist and neural engineer at Case Western Reserve University and at the US Department of Veterans Affairs Medical Center, both in Cleveland, Ohio. His research is mainly focused on implants that receive signals from the brain and which could also carry signals back into the brain or to other parts of the body. A key long-term aim of clinical researchers, including some at Case Western, is to restore motor function in people with paralysis and perhaps even to improve cognitive functions. Alzheimer's and multiple sclerosis are examples of possible diseases that might be targeted in this way.

When conventional, stiff

electrodes are implanted into the brain they can damage the tissues and cause inflammation. A flexible and biocompatible material would solve that problem, but it would be difficult to insert. "We needed something that was stiff to get it in but then softened once it was in," says Capadona. The sea-cucumber system suggested a model for a substrate that could surround a thin metal electrode and bring the necessary changes in rigidity to the combined structure.

Capadona and his colleagues developed a system of natural cellulose fibrils they call 'whiskers', which they combined with a synthetic polymer called polyvinyl acetate ${ }^{2}$. When dry, the material is rigid - the hydrogen bonds in the cross-links hold the cellulose in a semi-crystalline state - but when embedded in the brain it absorbs water and swells to become flexible. Capadona explains that fine control over the changes in stiffness and degree of swelling is crucial, rather than just a simple issue of changing between soft and stiff states. "This is why we looked to nature for a

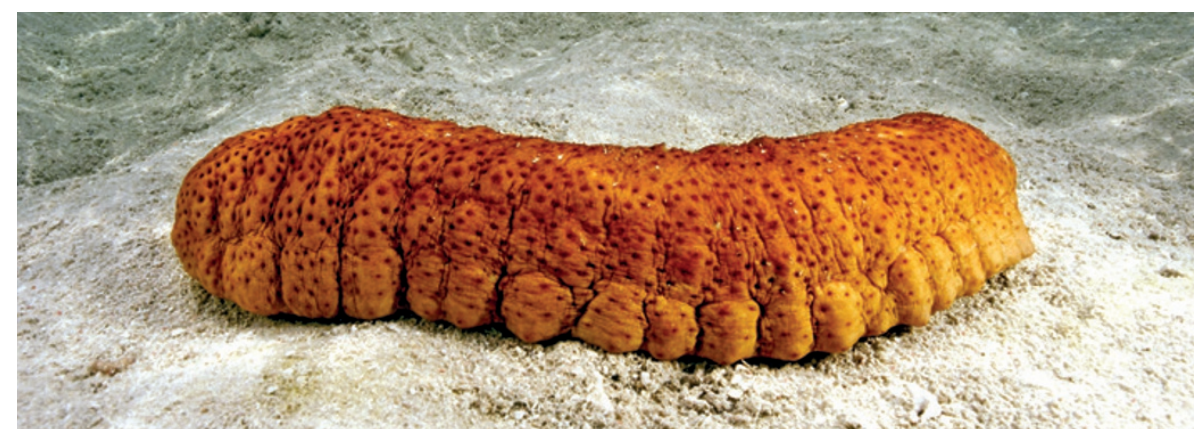

Studying sea cucumbers is helping researchers to develop advanced brain electrodes.

unique design to inspire ours," he says.

Tests of electrodes embedded in this material are being conducted on rats, with a goal of soon moving to trials on non-human primates and then eventually to humans. "It has proved to be one of the most successful approaches for minimizing tissue damage and inflammation," says Capadona of his most recent results, reporting negligible inflammation or loss of neurons ${ }^{3}$. So far, however, the only tests have been short term, involving rats under anaesthesia. Capadona says that his group must now undertake longer term studies.

Although he is focused mainly on developing functional electrodes for braincomputer interfaces, Capadona also envisages less sophisticated medical uses for the technology. Catheters and stents, for example, need to be rigid for insertion but flexibile once in place. His polymer-containing composite materials have even attracted some interest from toy manufacturers. He is not sure what applications toy designers might have in mind, but, he says, "It's fun to speculate."

\section{STRONG AND SMART HYDROGELS}

Much of the research into biomimetic polymers involves hydrogels - materials comprising networks of polymer chains that can absorb or release water as conditions change. Applications range from the mundane, such as high-absorbency diapers, to pioneering medical uses to help wounds to heal and biomedical implants to be accepted by the body. Fariba Dehghani, a bioengineer at the University of Sydney in Australia, has developed a biomimetic hydrogel based on natural gelatin and a mixture of synthetic polymers that could be used to regenerate bone $e^{4}$. In experiments in vitro, the hydrogel provides a home in which to grow bone cells that could be used to help repair fractures or rebuild limbs. The results of compression and strain tests have led Dehghani to claim that her material can withstand significant forces and should be assessed for bone-regeneration applications.

In addition to providing the substrate in which cells can live and grow, a key feature of many natural biological polymers is their ability to respond to a wide range of stimuli. In efforts to replicate this ability, some researchers are developing hydrogels that can mimic living tissues by swelling and then shrinking in response to chemical triggers and to changes in conditions such as temperature, $\mathrm{pH}$, electric and magnetic fields and light levels. Bioengineer Shyni Varghese of the Bio-Inspired Materials and Stem Cell Engineering Laboratory at the University of California, San Diego, says that in response to any of these stimuli, a hydrogel could be used to achieve multiple useful actions such as releasing a drug, opening and closing implanted valves and stents or sealing wounds.

"Nature has truly been an inspiration in designing stimuli-responsive hydrogels with dynamic functions and features," says Varghese, who co-authored a review of current and potential applications ${ }^{5}$. He emphasizes that most of the advances are still at the proof-ofconcept phase, but as an example of the technology already on the market he cites a class of temperature-responsive polymers known as poloxamers, which are moving into drugdelivery uses ${ }^{6}$. Varghese points to self-regulated insulin-delivery devices and hydrogel sealants for stomach perforations as applications that are moving out of the lab and into clinical use.

Conducting extensive real-world trials and gaining safety approvals are significant scientific challenges faced by many researchers on the path from laboratory to commercialization, but Wilker points out that having the correct technology is only part of the story. "You have to have the right personnel, the right landscape for competition and the right investors who put in enough money on a reasonable timeframe," he says. For Wilker and other researchers, the pieces are all coming together to make widespread use of biomimetics a reality. Years after his rough-but-inspiring scuba dive, Wilker hopes to take a dive into the turbulent waters of the marketplace very soon.

Andrew R. Scott is a science writer based in Perth, UK.

1. Meredith, H. J., Jenkins, C. L. \& Wilker, J. J. Adv Funct. Mater. 24, 3259-3267 (2014)

2. Jorfi, M., Skousen, J. L., Weder, C. \& Capadona, J. R. J. Neural. Eng. 12, 011001 (2015).

3. Nguyen, J. K. et al. J. Neural Eng. 11, 056014 (2014)

4. Fathi, A. et al. Eur. Polym. J. 59, 161-170 (2014)

5. Lim, L. H., Hwang, Y., Kar, M. \& Varghese, S. Biomater. Sci. 2, 603-618 (2014)

6. Shubhra, Q. T. H., Tóth, J., Gyenis, J. \& Feczkó, T. Polym. Rev. 54, 112-138 (2014). 